\title{
The determination of acidity of the dilute solutions of weak multibasic organic acids
}

\author{
Elene Kvaratskhelia, Ramaz Kvaratskhelia
}

R. Agladze Institute of Inorganic Chemistry and Electrochemistry, Tbilisi, Georgia.

Email: elicko@mail.ru; $\underline{\text { ekvarats@yahoo.com }}$

Received 12 February 2010; revised 1 March 2010, accepted 5 March 2010.

\begin{abstract}
The new theoretical method for the accurate determination of acidity of dilute solutions of weak multibasic organic acids (which are widely used in medicine, pharmacology, various branches of industry and participate in important biological processes in living organisms) is suggested. The concepts of the contributions of the separate dissociation steps to the $\left[H^{+}\right]$value, $x_{m}$, are used for an analysis of complex equilibria of the processes of dissociation of these acids. The cases of weak dibasic and tribasic organic acids with the "overlapping" dissociation equilibria and a general case of weak multibasic acids, $H_{n} A$, are considered. From the conditions of equality of the concentrations of various ionized and non-ionized forms in the dilute solutions of weak multibasic organic acids the areas of dominance of these forms in connection with the corresponding $x_{m}$ values are formulated.
\end{abstract}

Keywords: Dibasic Acids; Tribasic Acids; Hydrogen Ions Concentration; Equations

\section{INTRODUCTION}

Weak multibasic organic acids are widely used in medicine, pharmacology, chemical, food and cosmetic industries. Some of these acids participate in a series of important biological processes occurring in living organisms (for example, in the Krebs cycle). The majority of drugs are weak acids and/or bases. Their biopharmaceutical properties are directly connected with the dissociation constants and degrees of these compounds, consequently, with acidity of their solutions. The latter is the very factor which affects in physiological systems the rate at which the compound is able to diffuse across membranes and various obstacles, determines the acid-base homeostasis and enzyme kinetics in the cell and in the body. It is possible to say that an acidity of the weak multibasic organic acids determines, as a rule, all their useful (or harmful) properties.
Many weak multibasic organic acids have comparatively close values of the dissociation constants for the various steps; this fact causes their simultaneous participation in the determining the hydrogen ion concentration in solutions of these acids (i.e., "overlapping" dissociation equilibria). In this paper a new theoretical method for determination of acidity of the dilute (0.0001-0.1 $\mathrm{mol} \cdot \mathrm{dm}^{-3}$ ) solutions of such acids is suggested.

\section{RESULTS AND DISCUSSION}

\subsection{Dibasic Acids}

Dibasic acids form the most numerous group of weak multibasic organic acids with the "overlapping" equilibria effect. In dilute aqueous solutions the primary and secondary steps of dissociation are

$$
\begin{gathered}
H_{2} A \leftrightarrow H^{+}+H A^{-} \\
H A^{-} \leftrightarrow H^{+}+A^{2-}
\end{gathered}
$$

In our previous communications [1,2] we have used the concepts of the contributions to the total hydrogen ion concentration, $\left[\mathrm{H}^{+}\right]$, being assigned to the primary and secondary dissociation steps, $x_{1}$ and $x_{2}$, such that $x_{1}$ $+x_{2}=\left[H^{+}\right]$. The corresponding mass-action equations for both steps dissociation constants are

$$
\begin{gathered}
K_{1}=\frac{\left[H^{+}\right]\left(x_{1}-x_{2}\right)}{c-x_{1}} F_{l}=\frac{x_{1}^{2}-x_{2}^{2}}{c-x_{1}} F_{l} \\
K_{2}=\frac{\left[H^{+}\right] x_{2}}{x_{1}-x_{2}} F_{2}=\frac{x_{2}\left(x_{1}+x_{2}\right)}{x_{1}-x_{2}} F_{2}
\end{gathered}
$$

where $K_{1}$ and $K_{2}$ are the thermodynamic dissociation constants, c is the total (analytical) concentration of acid, $F_{1}$ and $F_{2}$ are the quotients of the activity coefficients:

$$
\begin{gathered}
F_{1}=\frac{f_{H^{+}} f_{H A^{-}}}{f_{H_{2} A}} \\
F_{2}=\frac{f_{H^{+}} f_{A^{2-}}}{f_{H A^{-}}}
\end{gathered}
$$


The values of the activity coefficients may be approximated by the Debye-Huckel equation:

$$
\log _{10} f_{i}=-\frac{z_{i}^{2} A \sqrt{I}}{1+a_{i} B \sqrt{I}}
$$

where $a_{i}$ is the cation-anion distance of closest approach, $A$ and $B$ are constants depending on the properties of water at given temperature, $z_{i}$ is the charge of ion. The ionic strength is given by $I=x_{1}+2 x_{2}$. The activity coefficient of undissociated acid is assumed to be unity.

According to the (3) and (4) the $x_{1}$ and $x_{2}$ values (and then their sum - the $\left[H^{+}\right]$value) can be calculated successively by an iterative solution of two quadratic equations:

$$
\begin{gathered}
x_{1}=\frac{1}{2}\left[-\frac{K_{1}}{F_{1}}+\sqrt{\left(\frac{K_{1}}{F_{1}}\right)^{2}+4\left(x_{2}^{2}+\frac{K_{1} c}{F_{1}}\right)}\right] \\
x_{2}=\frac{1}{2}\left[-\left(\frac{K_{2}}{F_{2}}+x_{1}\right)+\sqrt{\left.\left(\frac{K_{2}}{F_{2}}+x_{1}\right)^{2}+\frac{4 K_{2} x_{1}}{F_{2}}\right]}\right.
\end{gathered}
$$

We suggest also the empirical equation for the fast approximate determination of the $\mathrm{pH}$ values of dilute (0.0001-0.01 mol $\cdot \mathrm{dm}^{-3}$ ) solutions of weak dibasic (and tribasic with the low $\mathrm{K}_{3}$ values) organic acids:

$$
p H=-1.489+0.8 p K_{1}-\left(1.185-0.14 p K_{1}\right) \lg c
$$

The maximum value of the relative error for this equation for a series of weak dibasic and tribasic organic acids with the $p K_{1}$ values in the interval: 2.5-5 does not exceed $5 \%$ (the relative error is the ratio of the difference between the approximate $\mathrm{pH}$ value and corresponding accurate value, divided by the approximate $\mathrm{pH}$ value, and converted to percent).

\subsection{Tribasic Acids}

In case of weak tribasic organic acids with the "overlapping" dissociation equilibria, the mass-action equations may be expressed as follows:

$$
\begin{gathered}
K_{1}=\frac{\left[H^{+}\right]\left(x_{1}-x_{2}\right)}{c-x_{1}} F_{1}=\frac{\left(x_{1}+x_{2}+x_{3}\right)\left(x_{1}-x_{2}\right)}{c-x_{1}} F_{1} \\
K_{2}=\frac{\left[H^{+}\right]\left(x_{2}-x_{3}\right)}{x_{1}-x_{2}} F_{2}=\frac{\left(x_{1}+x_{2}+x_{3}\right)\left(x_{2}-x_{3}\right)}{x_{1}-x_{2}} F_{2} \\
K_{3}=\frac{\left[H^{+}\right] x_{3}}{x_{2}-x_{3}} F_{3}=\frac{\left(x_{1}+x_{2}+x_{3}\right) x_{3}}{x_{2}-x_{3}} F_{3}
\end{gathered}
$$

where

$$
F_{1}=\frac{f_{H^{+}} f_{H_{2} A^{-}}}{f_{H_{3} A}}
$$

$$
\begin{gathered}
F_{2}=\frac{f_{H^{+}} f_{H A^{2-}}}{f_{H_{2} A^{-}}} \\
F_{3}=\frac{f_{H^{+}} f_{A^{3-}}}{f_{H A^{2-}}}
\end{gathered}
$$

and $I=x_{1}+2 x_{2}+3 x_{3}$

The $x_{1}, x_{2}$ and $x_{3}$ values (and then their sum - the $\left[\mathrm{H}^{+}\right]$value) can be calculated successively by an iterative solution of three quadratic equations:

$$
\begin{aligned}
& x_{1}=\frac{1}{2}\left[-\left(\frac{K_{1}}{F_{1}}+x_{3}\right)+\sqrt{\left(\frac{K_{1}}{F_{1}}+x_{3}\right)^{2}+4\left(x_{2}^{2}+x_{2} x_{3}+\frac{K_{1} c}{F_{1}}\right)}\right] \\
& x_{2}=\frac{1}{2}\left[-\left(\frac{K_{2}}{F_{2}}+x_{1}\right)+\sqrt{\left(\frac{K_{2}}{F_{2}}+x_{1}\right)^{2}+4\left(x_{3}^{2}+x_{1} x_{3}+\frac{K_{2} x_{1}}{F_{2}}\right)}\right] \\
& x_{3}=\frac{1}{2}\left[-\left(\frac{K_{3}}{F_{3}}+x_{1}+x_{2}\right)+\sqrt{\left(\frac{K_{3}}{F_{3}}+x_{1}+x_{2}\right)^{2}+\frac{4 K_{3} x_{2}}{F_{3}}}\right]
\end{aligned}
$$

\subsection{Acids with the Higher Basicity}

It is necessary at first to consider the general case of the weak multibasic organic acid $H_{n} A$ with the "overlapping" dissociation equilibria. For this case we may write the equations connecting the values of $x_{1}, x_{2}$, $x_{3}, \ldots x_{n-1}, x_{n}$ with the concentrations of various anions: $x_{1}=\left[H_{n-1} A^{-}\right]+\left[H_{n-2} A^{2-}\right]+\left[H_{n-3} A^{3-}\right]+\ldots+\left[H A^{(n-1)-}\right]$

$$
\begin{aligned}
& x_{2}=\left[H_{n-2} A^{2-}\right]+\left[H_{n-3} A^{3-}\right]+\ldots+\left[H A^{(n-1)-}\right]+\left[A^{n-}\right] \\
& x_{3}=\left[H_{n-3} A^{3-}\right]+\ldots+\left[H A^{(n-1)-}\right]+\left[A^{n-}\right] \\
& x_{n-1}=\left[H A^{(n-1)-}\right]+\left[A^{n-}\right] \\
& x_{n}=\left[A^{n-}\right]
\end{aligned}
$$$$
+\left[A^{n-}\right](20)
$$

In a general form for the $\mathrm{m}$ dissociation step we may write:

$$
x_{m}=\left[H_{n-m} A^{m-}\right]+x_{m+1}
$$

The total hydrogen ion concentration may be expressed as follows:

$$
\left[\mathrm{H}^{+}\right]=\sum_{m=1}^{n} m\left[\mathrm{H}_{n-m} A^{\mathrm{m}-}\right]=\sum_{m=1}^{n} x_{m}
$$

The mass-action equation for the $m$ dissociation step may be expressed by the following equations:

$$
K_{m}=\frac{\left[H^{+}\right]\left(x_{m}-x_{m+1}\right)}{x_{m-1}-x_{m}} F_{m}=\frac{\left(x_{m}-x_{m+1}\right) \sum_{m=1}^{n} x_{m}}{x_{m-1}-x_{m}} F_{m}
$$

where 


$$
F_{m}=\frac{f_{H^{+}} f_{H_{n-m} A^{m-}}}{f_{H_{n-(m-1)^{A}}}}
$$

The equation for an ionic strength may be written as follows:

$$
I=\sum_{m=1}^{\mathrm{n}} m x_{m}
$$

In case of weak organic acids with the high (more than tribasic) basicity the conversion of (27) to the forms of $(8-9,17-19)$ leads to the very complicated expressions. That is why we suggest to solve the complicated problem of determining of acidity of the high-basic acids solutions by more simple method. Let us consider this method for the most difficult case of hexabasic mellitic (benzenehexacarboxylic) acid. First, assume that this acid can be treated as a tribasic acid (taking into account that the main contribution to the $\left[\mathrm{H}^{+}\right]$value is made by first three dissociation steps). Then the $x_{1}, x_{2}$ and $x_{3}$ values are determined successively by an iterative solution of (17-19), where the values of $F_{1}, F_{2}$ and $F_{3}$ were assumed to be unity. The obtained $x_{1}, x_{2}$ and $x_{3}$ values are then used for the determination of the initial estimate of $\left[\mathrm{H}^{+}\right]$. Then, with the aid of $\left[\mathrm{H}^{+}\right]$value and the iterative solution of the following equations (which are obtained from (27) for the corresponding dissociation steps):

$$
\begin{aligned}
& x_{4}=\frac{K_{4} x_{3}+\left[H^{+}\right] F_{4} x_{5}}{K_{4}+\left[H^{+}\right] F_{4}} \\
& x_{5}=\frac{K_{5} x_{4}+\left[H^{+}\right] F_{5} x_{6}}{K_{5}+\left[H^{+}\right] F_{5}} \\
& x_{6}=\frac{K_{6} x_{5}}{K_{6}+\left[H^{+}\right] F_{6}}
\end{aligned}
$$

the initial values of $x_{4}, x_{5}$ and $x_{6}$ are determined (assuming that $F_{4}, F_{5}$ and $F_{6}$ values to be unity). These values are used for a correction to the $\left[\mathrm{H}^{+}\right]$value:

$$
\left[\mathrm{H}^{+}\right]=\sum_{m=1}^{6} x_{m}
$$

and then obtaining the final (for this stage) $x_{4}, x_{5}$ and $x_{6}$ values. Then with the aid of the following equations (where $F_{4}, F_{5}$ and $F_{6}$ values are assumed to be unity):

$$
\begin{aligned}
& x_{1}=\frac{K_{1} c+\left[H^{+}\right] F_{1} x_{2}}{K_{1}+\left[H^{+}\right] F_{1}} \\
& x_{2}=\frac{K_{2} x_{1}+\left[H^{+}\right] F_{2} x_{3}}{K_{2}+\left[H^{+}\right] F_{2}} \\
& x_{3}=\frac{K_{3} x_{2}+\left[H^{+}\right] F_{3} x_{4}}{K_{3}+\left[H^{+}\right] F_{3}}
\end{aligned}
$$

improved $x_{1}, x_{2}$ and $x_{3}$ values are obtained. At the following stage, with the aid of the obtained six $x_{m}$ values, the ionic strength I is calculated with the aid of (29). The values of the activity coefficients of $\mathrm{H}^{+}$and all anions are approximated by the Debye-Huckel (7). With the aid of the activity coefficient values and (28) the $F_{1}, F_{2}, F_{3}, F_{4}, F_{5}$ and $F_{6}$ values are calculated. Using these values in (30) to (35), corrected values of all six $x_{m}$ values are obtained. With the aid of the latters, the final $\left[\mathrm{H}^{+}\right]$value is determined.

\subsection{The Use of the $X_{m}$ Concept for a Determination of the Concentrations of the Ionized and Non-Ionized Forms and their Distribution in the Dilute Solutions of Weak Multibasic Acids}

The determination of the $x_{m}$ values gives us also the opportunity to calculate the important dissociation parameters: the concentrations of all ionized and non-ionized forms of weak multibasic organic acids in their dilute solutions. For this goal in general case of $H_{n} A$ acid can be used (25) and (26). Taking into account also the following equation:

$$
\left[H_{n} A\right]=c-x_{1}
$$

We can formulate the conditions of an equality of the concentrations of ionized and non-ionized forms:

$$
\begin{aligned}
& {\left[H^{+}\right]=\left[H_{n} A\right]: c=2 x_{1}+\sum_{m=2}^{n} x_{m}} \\
& {\left[H_{n-1} A^{-}\right]=\left[H_{n} A\right]: c=2 x_{1}-x_{2}} \\
& {\left[H_{n-2} A^{2-}\right]=\left[H_{n} A\right]: c=x_{1}+x_{2}-x_{3}} \\
& {\left[H_{n-m} A^{m-}\right]=\left[H_{n} A\right]: c=x_{1}+x_{m}-x_{m+1}} \\
& {\left[A^{n-}\right]=\left[H_{n} A\right]: c=x_{1}+x_{n}}
\end{aligned}
$$

Taking into account these conditions, we may formulate the areas of dominance of various ionized and nonionized forms of acid:

$$
\left[H^{+}\right]>\left[H_{n} A\right]: c<2 x_{1}+\sum_{m=2}^{n} x_{m}
$$

(and vice versa)

$$
\left[H_{n-1} A^{-}\right]>\left[H_{n} A\right]: c<2 x_{1}-x_{2}
$$

(and vice versa)

$$
\left[H_{n-2} A^{2-}\right]>\left[H_{n} A\right]: c<x_{1}+x_{2}-x_{3}
$$

(and vice versa)

$$
\left[H_{n-m} A^{m-}\right]>\left[H_{n} A\right]: c<x_{1}+x_{m}-x_{m+1}
$$

(and vice versa)

$$
\left[A^{n-}\right]>\left[H_{n} A\right]: c<x_{1}+x_{n}
$$

(and vice versa) 


\section{CONCLUSIONS}

Many weak multibasic organic acids have the comparatively close values of the dissociation constants of the different steps. This fact causes the participation of all steps in formation the hydrogen ions total concentration in the solutions of such acids. We suggest the new theoretical method for a determination of acidity of these solutions using the concepts of the contributions of the separate dissociation steps to the $\left[H^{\dagger}\right]$ value, $x_{m}$. The equations for the accurate calculation of the $\left[H^{+}\right]$values in cases of dibasic and tribasic acids and also in general case of weak multibasic acid, $H_{n} A$, are suggested. The comparatively simple method for a determination of acidity of the dilute solutions of high-basic (more than tribasic) acids is also described. With the aid of the formulated by us conditions of an equality of the concentrations of various ionized and non-ionized forms in the dilute solutions of weak multibasic organic acids the areas of dominance of these forms in connection with the corresponding $x_{m}$ values are formulated.

\section{REFERENCES}

[1] Kvaratskhelia, E. and Kvaratskhelia, R. (2007) The degrees of dissociation of weak multibasic organic acids. Joural of Solution Chemistry, 36(6), 787-792.

[2] Kvaratskhelia, R. and Kvaratskhelia, E. (2000) Voltammetry of dicarboxylic acids on solid electrodes in aqueous and mixed media. Russian Journal of Electrochemisry, 36(3), 330-333. 